\title{
CYP2C19 genotype has prognostic value in specific populations following coronary stenting
}

\author{
Wenyao Wang ${ }^{1 \#}$, Chunli Shao ${ }^{1 \#}$, Bo Xu ${ }^{2}$, Jingjia Wang ${ }^{1}$, Min Yang ${ }^{1}$, Jing Chen ${ }^{1}$, Kuo Zhang ${ }^{1}$, \\ Siyuan Wang ${ }^{1}$, Ping Li ${ }^{1}$, Yi-Da Tang ${ }^{1,3}$
}

${ }^{1}$ Department of Cardiology, State Key Laboratory of Cardiovascular Disease, Fuwai Hospital, National Center for Cardiovascular Diseases, Chinese Academy of Medical Sciences and Peking Union Medical College, Beijing, China; ${ }^{2}$ Catheterization Laboratory, State Key Laboratory of Cardiovascular Disease, Fuwai Hospital, National Center for Cardiovascular Diseases, Chinese Academy of Medical Sciences and Peking Union Medical College, Beijing, China; ${ }^{3}$ Department of Cardiology and Institute of Vascular Medicine, Peking University Third Hospital, Key Laboratory of Molecular Cardiovascular Science, Ministry of Education, Beijing, China

Contributions: (I) Conception and design: YD Tang, P Li, W Wang; (II) Administrative support: C Shao, B Xu; (III) Provision of study materials or patients: B Xu; (IV) Collection and assembly of data: W Wang, J Wang, M Yang, J Chen, K Zhang, S Wang; (V) Data analysis and interpretation: W Wang; (VI) Manuscript writing: All authors; (VII) Final approval of manuscript: All authors.

\#These authors contributed equally to this work.

Correspondence to: Yi-Da Tang, MD, PhD. Department of Cardiology and Institute of Vascular Medicine, Peking University Third Hospital; Key Laboratory of Molecular Cardiovascular Science, Ministry of Education. No. 49 Huayuanbei Road, Beijing, China. Email: drtangyida@126.com; Ping Li, MD, PhD. Department of Cardiology, State Key Laboratory of Cardiovascular Disease, Fuwai Hospital, National Center for Cardiovascular Diseases, Chinese Academy of Medical Sciences and Peking Union Medical College, No. 167 Beilishi Road, Beijing 100037, China. Email: wklipingf@126.com.

Background: The prognostic value of the CYP2C19 genotype in post-percutaneous coronary intervention (PCI) patients remains controversial. The recently-published, limited-sample PHARMCLO trial indicates a personalized pharmacogenomic approach may reduce adverse events. This study aimed to determine the prognostic value of CYP2C19 genotypes.

Methods: The original cohort consisted of 10,724 PCI patients in 2013. 756 patients with genotyped CYP2C19 were included in our analysis. The CYP2C19 genotype prognostic value was tested based on different clinical factors. The primary endpoint was major adverse cardio- and cerebro-vascular event (MACCE).

Results: MACCE 2-years post-PCI occurred in 19 patients (17.4\%) in poor metabolizers (PM, CYP2C19 $\left.{ }^{*} 2 /{ }^{*} 2,{ }^{*} 2 /{ }^{*} 3,{ }^{*} 3 /{ }^{*} 3\right), 43$ patients $(12.2 \%)$ in intermediate metabolizers (IM, CYP2C19 * $1 /{ }^{*} 2$ or ${ }^{*} 1 /{ }^{*} 3$ ) and 27 patients $(9.2 \%)$ in extensive metabolizers (EM, CYP2C19*1/*1). PM was an independent MACCE predictor compared with EM (HR: 1.960, 95\% CI: 1.139-3.372), but the difference between IM and PM was not significant (HR: 1.314, 95\% CI: 0.843-2.048). Major bleeding (BARC grade $\geq 3$ ) was not significantly different between the three groups $(2.5 \%$ vs. $2.1 \%$ vs. $0.8 \%, \mathrm{P}=0.133)$. Subgroup analysis showed that the CYP2C19 genotype prognostic value was present in the following subgroups: male, age $>60$ years, body mass index $(\mathrm{BMI})>24 \mathrm{~kg} / \mathrm{m}^{2}$, SYNTAX score $>15$, current smokers, and patients without chronic kidney disease.

Conclusions: Utilizing CYP2C19 genotype to guide post-PCI antiplatelet therapy might be appropriate in patients with the following characteristics: male, age $>60$ years, BMI $>24 \mathrm{~kg} / \mathrm{m}^{2}$, SYNTAX score $>15$, current smokers, and non-chronic kidney disease (CKD) patients.

Keywords: Pharmacogenomics; antiplatelet; percutaneous coronary intervention; clopidogrel

Submitted Nov 29, 2020. Accepted for publication Mar 28, 2021.

doi: $10.21037 /$ atm-20-7724

View this article at: http://dx.doi.org/10.21037/atm-20-7724 


\section{Introduction}

Dual antiplatelet therapy (DAPT) with aspirin and P2Y12 inhibitors is the main approach to preventing ischemic events after the percutaneous coronary intervention (PCI) procedure (1). However, the most widely-used P2Y12 receptor inhibitor, clopidogrel, exhibits individual differences in efficacy (2). Genetic polymorphisms play an important role in individual drug reactions. Clopidogrel conversion to the active drug form is regulated by the CYP450 system, which presents multiple genetic polymorphisms (3). Numerous previous clinical pharmacogenomic studies determined that CYP2C19 lossof-function (LOF) alleles (including the *2 and *3 alleles) are associated with high on-treatment platelet reactivity (4-7). In addition, other genetic variants associated with clopidogrel metabolism (PON1 and $A B C B 1$ ) may also contribute to the individual antiplatelet efficacy of clopidogrel $(8,9)$.

Previous studies and meta-analyses showed that the CYP2C19 LOF allele in clopidogrel-treated PCI patients is associated with higher ischemic risk, including early stent thrombosis $(4,10-13)$. However, some other studies presented contradictory results (14-16). In addition to genetic polymorphisms, clopidogrel responses are also influenced by other factors. Some drugs, including proton pump inhibitors, statins, calcium channel blockers, and warfarin, could alter clopidogrel pharmacodynamics by competing for metabolic enzymes $(17,18)$. Clinical factors such as age, gender, diabetes, body mass index (BMI), renal dysfunction, and hyperlipidemia may also contribute to the individual clopidogrel response (19-22). How these factors comprehensively influence clopidogrel reactivity is of great significance.

The present study aimed to evaluate the prognostic value of CYP2C19 genotypes in patients with different clinical characteristics, including gender, age, BMI, synergy between percutaneous coronary intervention with taxus and cardiac surgery (SYNTAX) score, smoking status, diabetes mellitus, and chronic kidney disease. We provide evidence that the CYP2C19 genotype can inform optimal antiplatelet treatment with clopidogrel. We present the following article in accordance with the STROBE reporting checklist (available at http://dx.doi.org/10.21037/atm-20-7724).

\section{Methods}

\section{Study design and participants}

This is a retrospective, single-center cohort study.
The detailed study design is shown in Figure 1. The corresponding and first author had full access to all study data, and take responsibility for data integrity and analysis. The original cohort consisted of 10,724 PCI patients who were admitted to Fuwai Hospital, the National Center of Cardiovascular Diseases (Beijing, China), throughout 2013. Within this cohort, 756 patients taking CYP2C19 genotype test were eligible. Because ticagrelor was made available in the Chinese market at the end of 2012, only 4 subjects in our original cohort used this medication to replace clopidogrel. Another 9 patients took doubledosage clopidogrel or triple-antiplatelet medication with cilostazol before discharge. These 12 patients were excluded from our analysis because different antiplatelet strategies have confounding effects on the predictive value of CYP2C19 genotypes. Two-year follow-up was completed after PCI with either a subsequent visit or by telephone. Exclusion criteria included aspirin or clopidogrel allergy, bleeding disorder, chronic oral anticoagulation drugs such as warfarin, contraindication to antiplatelet therapy, severe anemia, tumor, or severe immune system disorder. Baseline information and clinical profiles were retrieved from medical records. Chronic kidney disease (CKD) was defined as estimated glomerular filtration rate (eGFR) $<60 \mathrm{~mL} / \mathrm{min} / 1.73 \mathrm{~m}^{2}$ for $\geq 3$ months, with or without kidney damage. Diabetes mellitus was defined as at least $200 \mathrm{mg} / \mathrm{dL}$ blood glucose after a 2-hour glucose tolerance test, fasting glucose $\geq 126 \mathrm{mg} / \mathrm{dL}$ ( $\geq 7.0 \mathrm{mM}$ ), HbA1c $\geq 6.5 \%$, physiciandiagnosed diabetes, and/or use of diabetic medication.

All the participants provided signed informed consent for the original cohort study and subsequent analysis. The study was conducted in accordance with the Declaration of Helsinki (as revised in 2013). The study was approved by institutional ethics committee of Fuwai Hospital (No. 2016847 ) and individual consent for this retrospective analysis was waived.

\section{DNA extraction and CYP2C19 genotyping}

CYP2C19 genotypes were tested by gene sequencing in the Central Laboratory of Fuwai Hospital during hospitalization. We extracted DNA from peripheral blood with a Thermo Kingfisher Flex system (BOKUN BIOTECH, China). DNA was stored at $-80{ }^{\circ} \mathrm{C}$ until analysis. CYP2C19*2 and *3 were genotyped using a CYP2C19*2 and *3 Gene Detection Fluorescence PCR Capillary Electrophoresis Sequencing Analysis Kit (Beijing SinoMDgene Technology Co., Ltd) and was performed on 


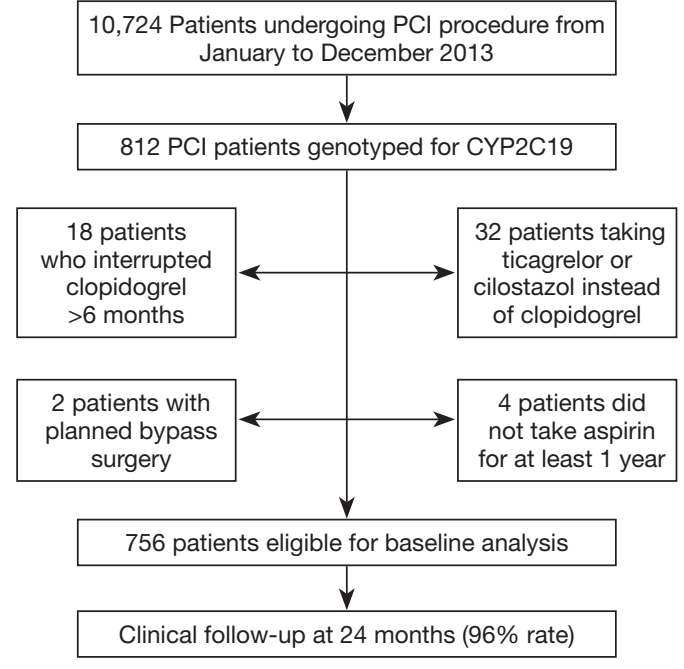

Figure 1 Study flow diagram.

an ABI 3500xL Dx DNA Analyzer (Applied Biosystems, Foster City, CA, USA). Genotypes were confirmed independently by two professionals. $5 \%$ of sequence data was verified by resequencing. CYP2C19*2 and *3 alleles were defined as "LOF" alleles. Patients with ${ }^{*} 1 /{ }^{*} 1$ alleles were defined as extensive metabolizers $(\mathrm{EM})$, those with a single ${ }^{*} 2$ or ${ }^{*} 3$ allele (i.e., ${ }^{*} 1 /{ }^{*} 2$ or ${ }^{*} 1 /{ }^{*} 3$ ) were defined as intermediate metabolizers (IM), and those with two *2 or *3 alleles (i.e., ${ }^{*} 2 /{ }^{*} 2,{ }^{*} 2 /{ }^{*} 3$ or ${ }^{*} 3 /{ }^{*} 3$ ) were defined as poor metabolizers (PM).

\section{Antiplatelet treatment, follow-up, study endpoints, and subgroup analysis strategy}

All 756 post-PCI patients received a $300 \mathrm{mg}$ loading dose of clopidogrel and aspirin before PCI, followed by a daily $100 \mathrm{mg}$ maintenance dose of aspirin and $75 \mathrm{mg}$ clopidogrel for at least 1 year. Clinical and telephone follow-up was conducted on Day 30 and Months 6, 12, and 24 to monitor ischemic and bleeding endpoints.

The primary endpoint of our study was major adverse cardiovascular and cerebrovascular event (MACCE, a composite of all-cause death, myocardial infarction, target vessel revascularization, and cerebrovascular events). An independent event committee of two cardiologists was responsible for events adjudication. Academic Research Consortium (ARC) (23)-defined stent thrombosis was also recorded. Major bleeding was defined as Bleeding Academic Research Consortium (BARC) (24) type 3 and 5 bleeding events. Acute myocardial infarction was diagnosed in accordance with the universal definition proposed in 2012. Target vessel revascularization was clinically driven. Stroke was defined as focal neurologic function loss caused by an ischemic or hemorrhagic event, with residual symptoms lasting at least 24 hours or leading to death.

Variables included in the multivariate Cox analysis and used for subgroup stratification were selected by reviewing previous studies about potential factors contributing to the individual clopidogrel response. Finally, we took gender, age, BMI, SYNTAX score, smoking status, diabetes mellitus, and chronic kidney disease into the multivariate model. The threshold of age was selected based on the WHO definition of old age (60 years). BMI $>24 \mathrm{~kg} / \mathrm{m}^{2}$ is according to the Chinese overweight standard. SYNTAX tertiles were used to define high-risk (upper tertile, $>15$ ) and intermediate-low risk groups (medium-lower tertile, $\leq 15$ ).

\section{Statistical analysis}

All continuous variables are presented as mean \pm SD. Analysis of variance (ANOVA) was used to compare means across multiple groups. Non-continuous and categorical variables are presented as frequencies or percentages and were compared using Chi-square tests or Fisher's exact tests. Kaplan-Meier curve analysis was used to calculate time to clinical endpoints. Between-group differences were evaluated by log-rank tests. The Cox proportional hazards model was further applied to estimate hazard ratios, and the proportional hazards assumptions were tested by $\log$ minus log plot. All patient data were censored at the date of the last available information. Cox analysis was performed to establish clinical variables associated with clinical events. The interaction between CYP2C19 genotypes and clinical risk factors was tested using Cox analysis. A two-sided $\mathrm{P}$ value $<0.05$ indicated statistical significance. Statistical analysis was performed using SPSS version 21 software (IBM Institute Inc., USA).

\section{Results}

\section{Baseline study population characteristics}

From January to December 2013, we recruited 756 eligible PCI patients and determined their CYP2C19 genotypes. All the patients included in the present study underwent PCI procedure with $2^{\text {nd }}$ generation DES (new-generation DES). The utilization of intravenous ultrasound imaging 
Table 1 Baseline study population characteristics stratified by CYP2C19 genotype

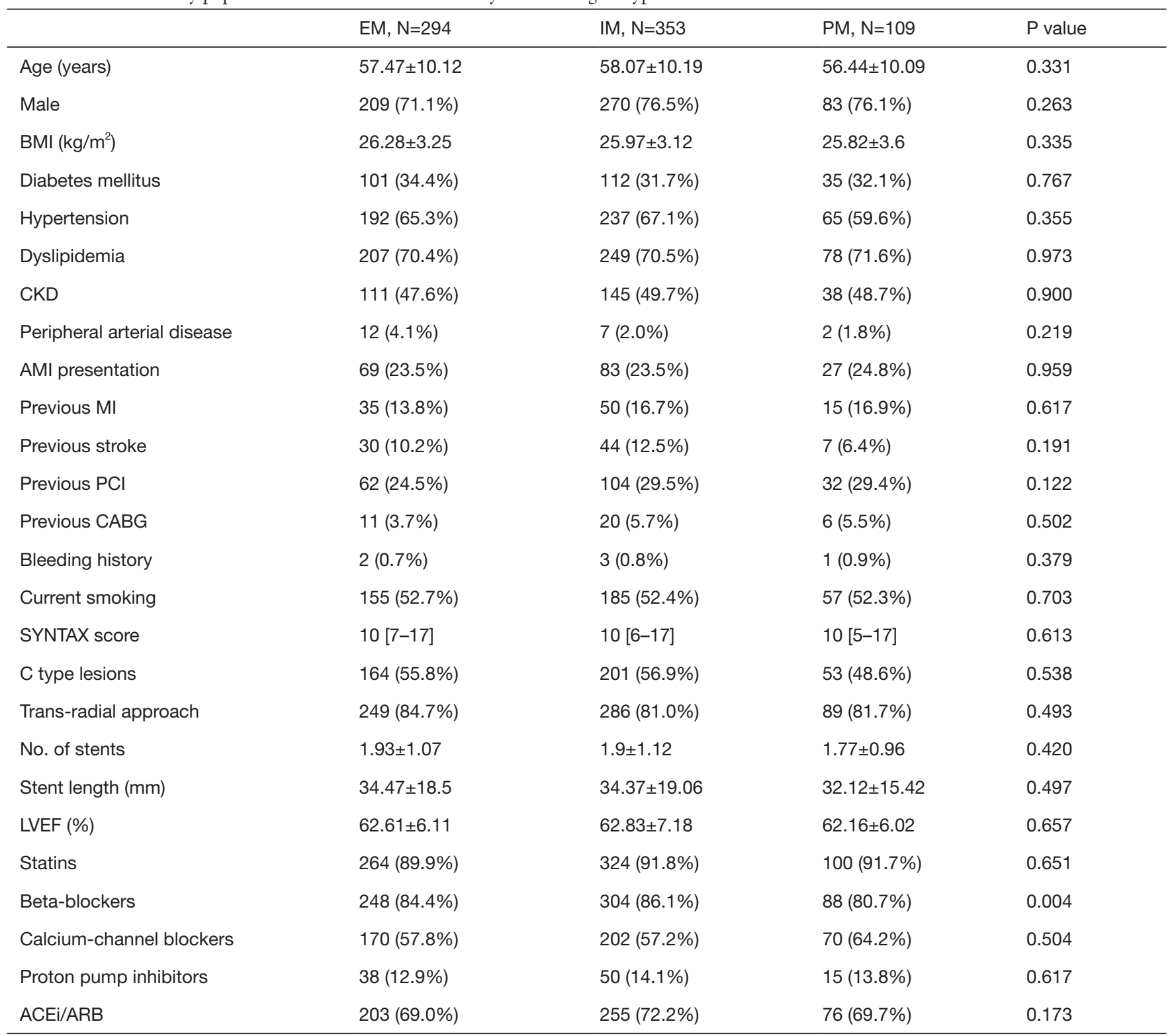

Values are mean \pm SD or $n(\%)$. Continuous variables were compared using one-way ANOVA and Bonferroni post-hoc analysis. Categorical variables were compared using Chi-square test. EM, extensive metabolizers, CYP2C19 genotype * $1 /{ }^{\star} 1$; IM, intermediate metabolizers, CYP2C19 genotype * $1 /{ }^{*} 2$ or ${ }^{*} 1 /{ }^{*} 3$; PM, poor metabolizers, CYP2C19 genotype *2/*2, ${ }^{*} 2{ }^{\star} 3$, or ${ }^{\star} 3 /{ }^{\star} 3$; BMI, body mass index; $\mathrm{CKD}$, chronic kidney disease; MI, myocardial infarction; $\mathrm{PCl}$, percutaneous coronary intervention; CABG, coronary artery bypass graft; LVEF, left ventricular ejection fraction; ACEi, angiotensin-converting enzyme inhibitor; ARB, angiotensin receptor blocker.

could be found in about $10 \%$ in the whole cohort. Based on CYP2C19 genotyping results, 294 patients (38.9\%) were included in the EM group (i.e., $\left.{ }^{*} 1 /{ }^{*} 1\right), 353$ patients $(46.7 \%)$ in the IM group (i.e., ${ }^{*} 1 /{ }^{*} 2$ or $\left.{ }^{*} 1 /{ }^{*} 3\right)$, and 109 patients $(14.4 \%)$ in the PM group (i.e., ${ }^{*} 2 /{ }^{*} 2,{ }^{*} 2 / * 3$ or $* 3 / * 3)$. During the 2 -year follow-up period, adverse event and vital status information was available for $96 \%$ of the total study population. Baseline subject characteristics are presented in Table 1. Most comorbidities, medications, and PCI procedure parameters were well matched, except for beta-blocker use $(84.4 \%$ in $\mathrm{EM}, 86.1 \%$ in IM, and $80.7 \%$ in $\mathrm{PM}, \mathrm{P}=0.004)$. The transradial approach was used in 
Table 2 Univariate Cox survival analysis for MACCE events among different CYP2C19 genotypes

\begin{tabular}{|c|c|c|c|c|c|c|}
\hline & \multicolumn{3}{|c|}{ IM vs. EM } & \multicolumn{3}{|c|}{ PM vs. EM } \\
\hline Whole cohort & 1.314 & $0.843-2.048$ & 0.227 & 1.960 & $1.139-3.372$ & 0.015 \\
\hline Male & 1.394 & $0.827-2.351$ & 0.213 & 2.068 & 1.098-3.895 & 0.024 \\
\hline Female & 1.105 & $0.469-2.603$ & 0.819 & 1.676 & $0.572-4.906$ & 0.346 \\
\hline SYNTAX score $\leq 15$ & 1.071 & $0.595-1.928$ & 0.819 & 1.342 & $0.611-2.948$ & 0.463 \\
\hline Age $>60$ & 1.353 & $0.682-2.687$ & 0.387 & 2.736 & $1.248-5.998$ & 0.012 \\
\hline Age $\leq 60$ & 1.284 & $0.717-2.300$ & 0.400 & 1.461 & $0.679-3.142$ & 0.332 \\
\hline $\mathrm{BMI}>24$ & 1.525 & $0.902-2.578$ & 0.115 & 2.648 & $1.390-5.042$ & 0.003 \\
\hline Non-DM & 1.222 & $0.705-2.119$ & 0.476 & 1.858 & $0.945-3.654$ & 0.073 \\
\hline Current smoking & 1.415 & $0.775-2.586$ & 0.259 & 2.024 & $0.967-4.238$ & 0.062 \\
\hline Non-smoking & 1.212 & $0.628-2.336$ & 0.567 & 1.887 & $0.847-4.200$ & 0.120 \\
\hline CKD & 0.960 & $0.466-1.977$ & 0.912 & 1.350 & $0.513-3.552$ & 0.543 \\
\hline Non-CKD & 1.603 & $0.911-2.818$ & 0.101 & 2.372 & $1.219-4.613$ & 0.011 \\
\hline
\end{tabular}

EM, extensive metabolizers, CYP2C19 genotype * $1 /{ }^{*} 1$; IM, intermediate metabolizers, CYP2C19 genotype * $1 /{ }^{*} 2$ or * $1 /{ }^{*} 3$; PM, poor metabolizers, CYP2C19 genotype ${ }^{*} 2 /{ }^{*} 2,{ }^{*} 2 /{ }^{*} 3$, or ${ }^{*} 3 /{ }^{*} 3$; BMI, body mass index; CKD, chronic kidney disease; MI, myocardial infarction; DM, diabetes mellitus.

$84.7 \%, 81.0 \%$, and $81.7 \%$ of patients in the three groups, respectively $(\mathrm{P}=0.493)$. Total stent length per patient was $34.47 \pm 18.5,34.37 \pm 19.06$, and $32.12 \pm 15.42 \mathrm{~mm}$, respectively $(\mathrm{P}=0.497)$. Acute myocardial infarction occurrence at admission was not statistically different $(23.5 \%, 23.5 \%$, and $24.8 \% ; \mathrm{P}=0.959)$. Similar SYNTAX scores were found among the three groups $(\mathrm{P}=0.613)$.

\section{Clinical outcomes in the whole cohort}

The primary endpoint of MACCE events (defined as a composite of all-cause death, myocardial infarction, target vessel revascularization or stroke) two years postPCI occurred in 19 patients (17.4\%) in the PM group, 43 patients $(12.2 \%)$ in the IM group, and 27 patients (9.2\%) in the EM group. In univariate Cox analysis, PM was an independent predictor for MACCE events compared with EM (see Table 2; HR: 1.960, 95\% CI: 1.139-3.372, $\mathrm{P}=0.015)$, but the difference between the IM and PM groups was not significant (see Table 2; HR: 1.314, 95\% CI: $0.843-2.048, \mathrm{P}=0.227$ ). After adjusting for potential confounders including age, gender, diabetes, body mass index, renal dysfunction, smoking, and SYNTAX score, $\mathrm{PM}$ was still the most significant MACCE predictor (see Table 3; HR: 1.957, 95\% CI: 1.132-3.384, $\mathrm{P}=0.016$ ). Again, no significantly increased adverse event risk was detected between PM and EM groups (HR: 1.344, 95\% CI: 0.861$2.098, \mathrm{P}=0.194)$. Figure 2 shows the cumulative KaplanMeier MACCE estimates among the three groups (PM vs. EM: log-rank $\mathrm{P}=0.014$; IM vs. EM: log-rank $\mathrm{P}=0.223$ ).

No significant difference was detected in the major bleeding rates (BARC grade $\geq 3$ ) between the three groups (2.1\% in PM, $2.3 \%$ in IM, and 2.6\% in EM). Regarding instent thrombosis, the very low incidence in the study cohort might explain the lack of significant differences. There was 1 case $(0.9 \%)$ in PM, 6 cases (1.7\%) in IM, and 2 cases $(0.7 \%)$ in $\mathrm{EM}$.

\section{Subgroup analysis of CYP2C19 genotype prognostic value}

To evaluate the prognostic value of CYP2C19 genotypes in patients with different clinical characteristics, subgroup 
Table 3 Multivariate Cox survival analysis for MACCE events among different CYP2C19 genotypes

\begin{tabular}{|c|c|c|c|c|c|c|}
\hline & \multicolumn{3}{|c|}{ IM vs. EM } & \multicolumn{3}{|c|}{ PM vs. EM } \\
\hline & $\mathrm{HR}$ & $95 \% \mathrm{Cl}$ & $P$ value & $\mathrm{HR}$ & $95 \% \mathrm{Cl}$ & $P$ value \\
\hline Whole cohort & 1.344 & $0.861-2.098$ & 0.194 & 1.957 & $1.132-3.384$ & 0.016 \\
\hline Male & 1.434 & $0.849-2.422$ & 0.178 & 2.067 & $1.087-3.391$ & 0.027 \\
\hline Female & 1.145 & $0.480-2.729$ & 0.760 & 1.447 & $0.481-4.353$ & 0.511 \\
\hline SYNTAX score $>15$ & 1.717 & $0.861-3.423$ & 0.125 & 2.952 & $1.329-6.558$ & 0.008 \\
\hline Age $>60$ & 1.380 & $0.693-2.749$ & 0.360 & 2.721 & $1.215-6.093$ & 0.015 \\
\hline Age $\leq 60$ & 1.278 & $0.711-2.298$ & 0.412 & 1.585 & $0.726-3.462$ & 0.248 \\
\hline $\mathrm{BMI}>24$ & 1.566 & $0.923-2.657$ & 0.096 & 2.391 & $1.249-4.577$ & 0.008 \\
\hline $\mathrm{BMI} \leq 24$ & 0.947 & $0.405-2.216$ & 0.901 & 0.977 & $0.343-2.781$ & 0.965 \\
\hline Current smoking & 1.462 & $0.798-2.678$ & 0.218 & 2.153 & $1.017-4.560$ & 0.045 \\
\hline Non-smoking & 1.199 & $0.619-2.323$ & 0.591 & 1.819 & $0.801-4.132$ & 0.153 \\
\hline CKD & 0.972 & $0.470-2.012$ & 0.939 & 1.078 & $0.397-2.923$ & 0.883 \\
\hline Non-CKD & 1.613 & $0.915-2.845$ & 0.098 & 2.412 & $1.231-4.727$ & 0.010 \\
\hline
\end{tabular}

EM, extensive metabolizers, CYP2C19 genotype ${ }^{*} 1 /{ }^{*} 1$; IM, intermediate metabolizers, CYP2C19 genotype * $1 /{ }^{*} 2$ or ${ }^{*} 1 /{ }^{*} 3$; PM, poor metabolizers, CYP2C19 genotype ${ }^{*} 2 /{ }^{*} 2,{ }^{\star} 2 /{ }^{*} 3$, or ${ }^{*} 3 /{ }^{*} 3$; BMI, body mass index; CKD, chronic kidney disease; MI, myocardial infarction; DM, diabetes mellitus.

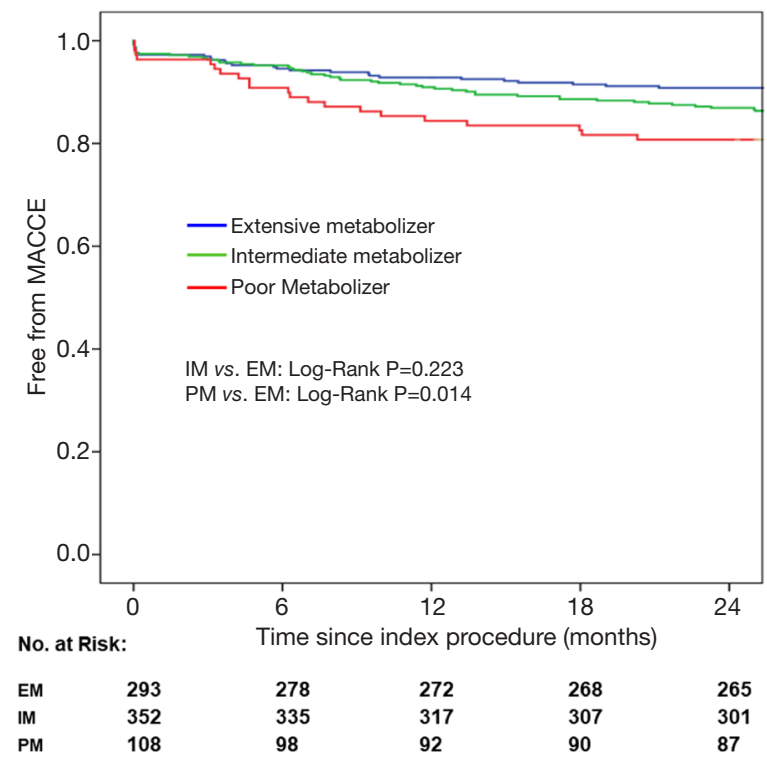

Figure 2 Cumulative Kaplan-Meier estimates of the primary endpoints (MACCE) in the whole cohort. MACCE, major adverse cardiac and cerebrovascular events. EM, extensive metabolizers, CYP2C19 genotype * $1 /{ }^{*} 1$; IM, intermediate metabolizers, CYP2C19 genotype ${ }^{*} 1 /{ }^{*} 2$ or ${ }^{*} 1 /{ }^{*} 3$; PM, poor metabolizers, CYP2C19 genotype *2/*2, *2/*3, or *3/*3. analysis was performed at the following levels: male or female, age $>60$ years or $\leq 60$ years, BMI $>24 \mathrm{~kg} / \mathrm{m}^{2}$ or $\leq 24 \mathrm{~kg} / \mathrm{m}^{2}$, SYNTAX score $>15$ or $\leq 15$, smoking or non-smoking, diabetes mellitus (DM) or non-DM. The multivariate Cox analysis presented in Table 3 was also adjusted for the above confounders.

The prognostic value of the PM genotype, which has been detected in the whole cohort, differed in patients with different characteristics (Tables 2,3). A significant CYP2C19 genotype prognostic value (PM vs. EM) was found in the following subgroups (Figure 3): male (HR: 2.067, 95\% CI: 1.087-3.391, $\mathrm{P}=0.027$ ), age $>60$ years (HR: $2.721,95 \% \mathrm{CI}$ : 1.215-6.093, $\mathrm{P}=0.015$ ), $\mathrm{BMI}>24 \mathrm{~kg} / \mathrm{m}^{2}$ (HR: $2.391,95 \%$ CI: 1.249-4.577, P=0.008), SYNTAX score >15 (HR: 2.952, 95\% CI: 1.329-6.558, $\mathrm{P}=0.008)$, current smoking (HR: 2.153, 95\% CI: $1.017-4.560, \mathrm{P}=0.045)$, and non-CKD (HR: 2.412, 95\% CI: $1.231-4.727, \mathrm{P}=0.010)$. In patients without these clinical characteristics, no significant correlation was found between CYP2C19 genotype and clinical prognosis. Figures 4 and 5 show the cumulative Kaplan-Meier estimates of MACCE in different subgroups. Interestingly, the CYP2C19 prognostic value (PM vs. EM) was similar 


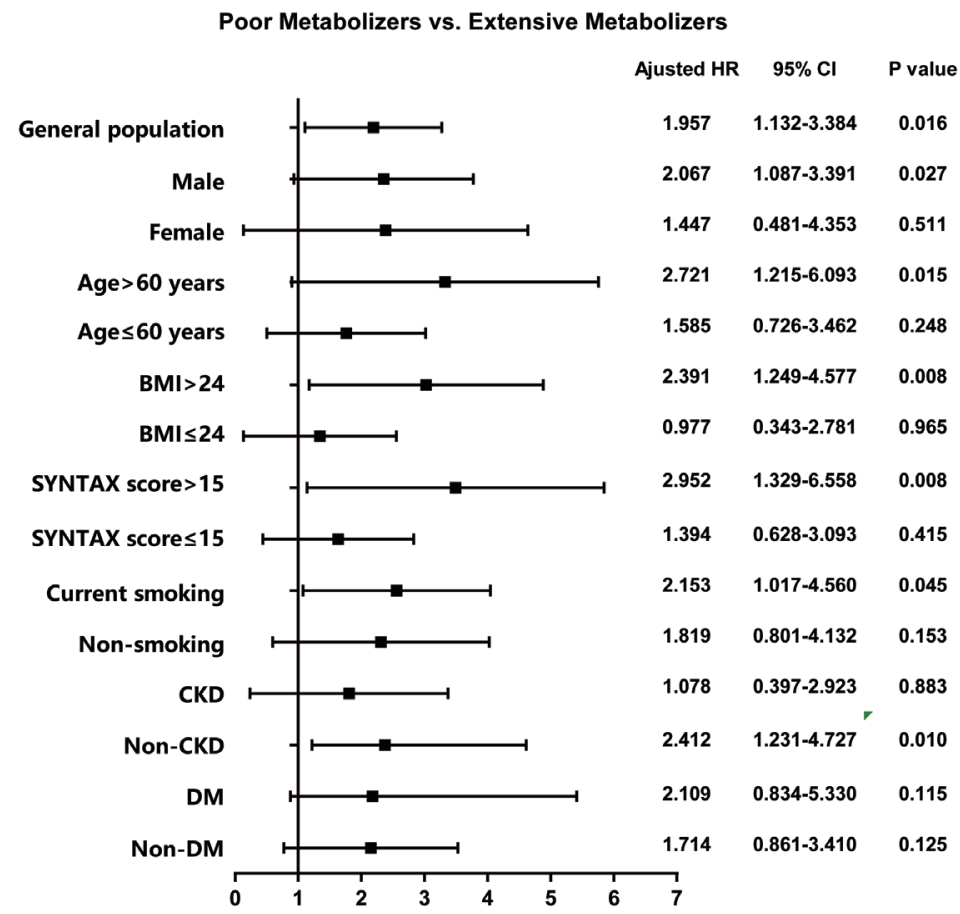

Figure 3 Hazard ratios from CYP2C19 genotype prognostic value subgroup analysis. Extensive metabolizers, CYP2C19 genotype * $1 /{ }^{*} 1$; poor metabolizers, CYP2C19 genotype *2/*2, *2/*3, or *3/*3; BMI, body mass index; CKD, chronic kidney disease; DM, diabetes mellitus.

in patients with DM (HR: 2.109, 95\% CI: 0.834-5.330, $\mathrm{P}=0.115$ ) or without DM (HR: 1.714, 95\% CI: 0.861-3.410, $\mathrm{P}=0.125)$.

\section{Discussion}

The prognostic value of CYP2C19 genotypes in postPCI patients is still controversial. A recently-published study (25) named the PHARMCLO trial indicated that personalized pharmacogenomic approaches may reduce adverse events. However, the study was only conducted in a limited sample population. Our results suggest that the CYP2C19 predictive value manifests in patients with the following characteristics: male, age $>60$ years, BMI $>24 \mathrm{~kg} /$ $\mathrm{m}^{2}$, SYNTAX score $>15$, current smokers, and non-CKD. These findings support the idea that utilizing the CYP2C19 genotype to guide post-PCI antiplatelet therapy in specific populations might be appropriate.

It was previously reported that platelet aggregation increases gradually when haplotypes * $1,{ }^{*} 2$, and *3 were replicated by diplotypes (3). CYP2C19 LOF alleles *2 and ${ }^{*} 3$ are all associated with platelet aggregation risk. Similarly, platelet aggregation activity showed a slight uptrend along with increased LOF alleles. However, it was also reported that clopidogrel responses can be influenced by other factors. The other two genetic variants associated with clopidogrel metabolism (PON1 and $A B C B 1$ ) could contribute to the individual antiplatelet efficacy of clopidogrel $(8,9)$. Some drugs, including proton pump inhibitors, statins, calcium channel blockers, warfarin, and others, could alter the clopidogrel pharmacodynamics by competing with metabolic enzymes $(17,18)$. Clinical factors such as age, gender, diabetes, BMI, renal dysfunction, and hyperlipidemia may also contribute to the individual clopidogrel response $(11,19,20)$. With complex confounding factors, previous studies evaluating the prognostic value of CYP2C19 did not present a convincing conclusion to guide clinical applications.

In early 2020, Angiolillo DJ and his colleagues published the development and validation of ABCD-GENE score, which is a useful tool to identify patients at high risk for high on-treatment platelet reactivity (26). Combining 4 clinical factors including age, BMI, CKD and diabetes and 1 genetic factor (CYP2C19 LOF), the ABCD-GENE score was validated in the external cohorts, including a Japan cohort (27). These findings supported the view derived 

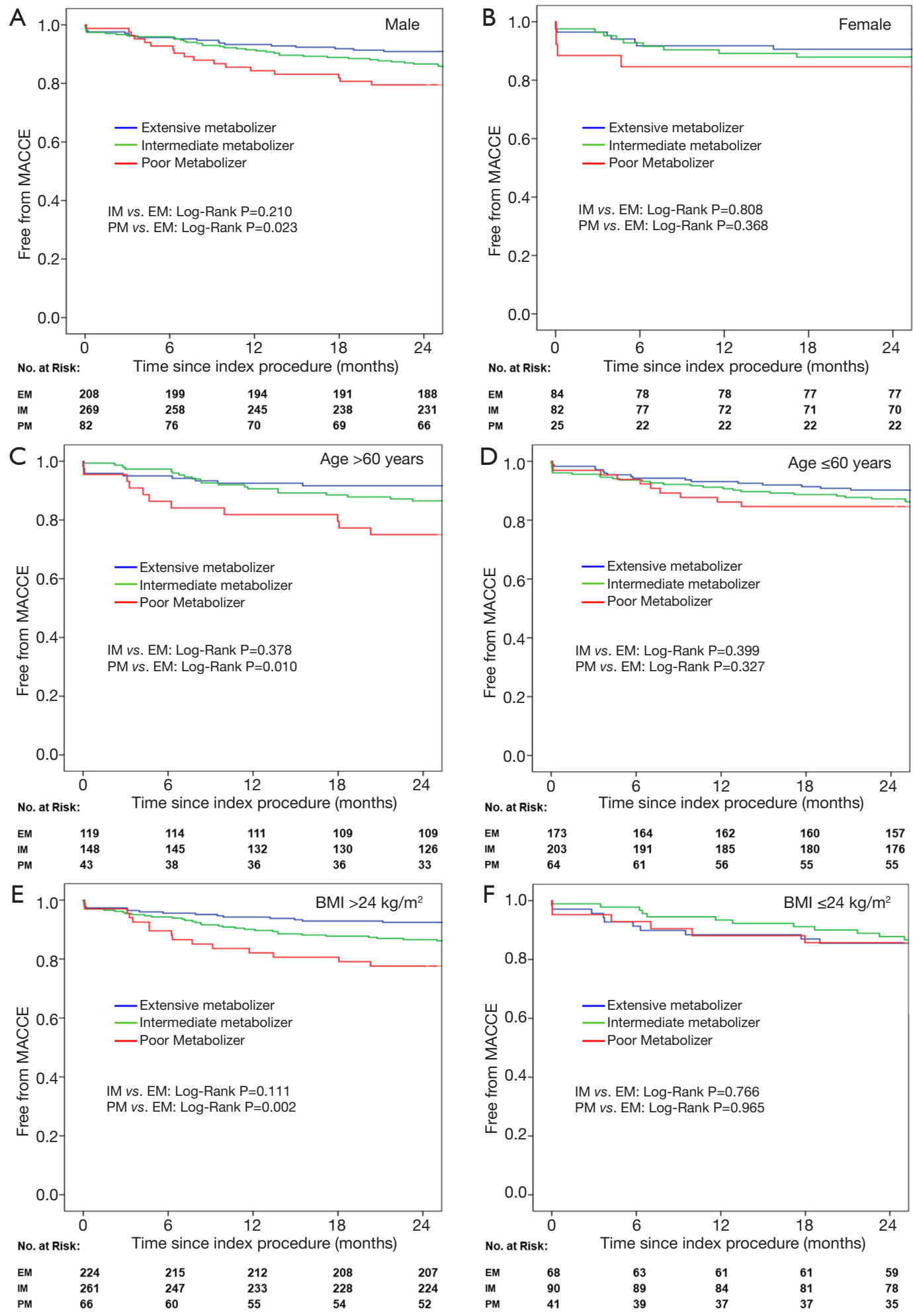

Figure 4 Cumulative Kaplan-Meier MACCE Estimates in Sex, Age, and BMI Subgroups. (A) male subgroup; (B) female subgroup; (C) age $>60$ years subgroup; (D) age $\leq 60$ years subgroup; (E) BMI $>24 \mathrm{~kg} / \mathrm{m}^{2}$ subgroup; (F) BMI $\leq 24 \mathrm{~kg} / \mathrm{m}^{2}$ subgroup. EM, extensive metabolizers, CYP2C19 genotype *1/*1; IM, intermediate metabolizers, CYP2C19 genotype *1/*2 or *1/*3; PM, poor metabolizers, CYP2C19 genotype ${ }^{*} 2 / * 2,{ }^{*} 2 / 3$, or $* 3 / * 3$. 

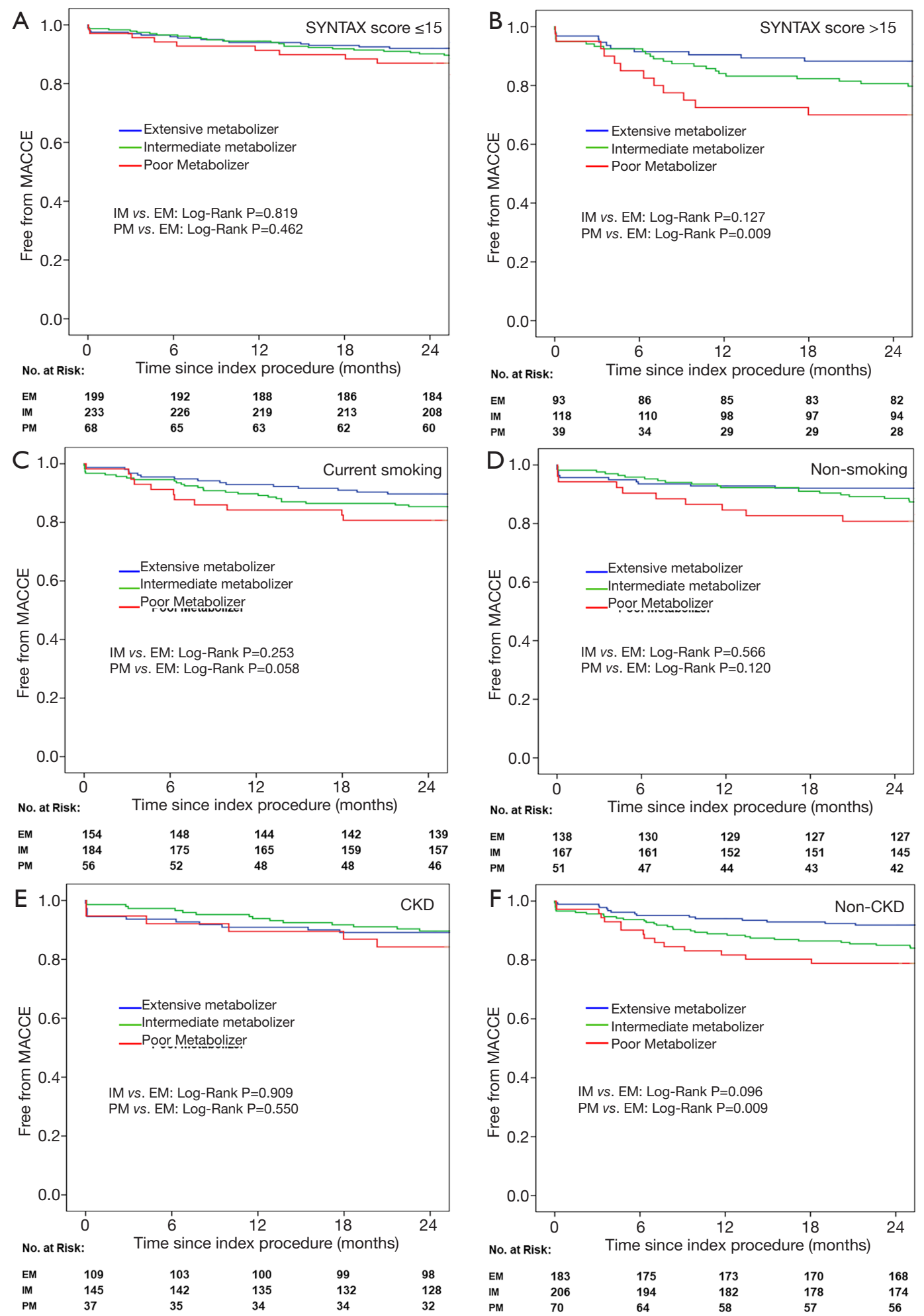

Figure 5 Cumulative Kaplan-Meier MACCE Estimates in SYNTAX score, smoking, and CKD subgroups. (A) SYNTAX score $\leq 15$ subgroup; (B) SYNTAX score >15 subgroup; (C) current smoking subgroup; (D) non-smoking subgroup; (E) CKD subgroup; (F) non-CKD subgroup. EM, extensive metabolizers, CYP2C19 genotype * $1 /{ }^{*} 1$; IM, intermediate metabolizers, CYP2C19 genotype * $1 /{ }^{*} 2$ or * $1 /{ }^{*} 3$; PM, poor metabolizers, CYP2C19 genotype ${ }^{*} 2 /{ }^{*} 2,{ }^{*} 2 / * 3$, or $* 3 / * 3$. 
from our study: CYP2C19 genotype should be interpreted and applicated based on specific clinical settings. In recent years, two large-scale clinical trials of CYP2C19 genotypeguided antiplatelet therapy following percutaneous coronary intervention offer new insight of the clinical utility of genotyping strategy to personalize antiplatelet therapy selection. The POPular Genetics trial published in 2019 found that CYP2C19 genotype-guided strategy for selection of oral P2Y12 inhibitor therapy was noninferior to standard treatment with ticagrelor or prasugrel at 12 months with respect to thrombotic events and resulted in a lower incidence of bleeding (28). In the TAILOR-PCI trial published in 2020, among CYP2C19 LOF carriers undergoing PCI, genotype-guided selection of an oral P2Y12 inhibitor, compared with conventional clopidogrel therapy, resulted in no statistically significant difference in a composite ischemic endpoint (29). The above two trials indicated that the separate utilization of CYP2C19 genotype in general PCI population might not be reasonable.

In the PHARMCLO trial, researchers evaluated whether selecting antiplatelet therapy (clopidogrel, prasugrel, or ticagrelor) on the basis of patient genetic and clinical characteristics leads to better clinical outcomes. Although the study was prematurely terminated because of lacking in vitro diagnosis certification, this trial still suggests improved outcomes after evaluation of genetic factors. An obviously different strategy taken in this study is that although all the patients in the pharmacogenomic group received genotyping tests, the final decision was left to doctors after comprehensively balancing ischemic/bleeding risk. Taking clinical characteristics into account, pharmacogenomic information became an important decision-making factor. The further questions are: (I) Is pharmacogenomic guidance applicable for all PCI patients? and (II) If not, who should receive pharmacogenomic testing? Our study provides preliminary indications for these questions.

Bliden et al. reported that current smokers on clopidogrel therapy displayed significantly lower platelet aggregation and adenosine diphosphate (ADP)-stimulated active glycoprotein (GP) IIb/IIIa expression compared with non-smokers (30), indicating that smoking might be an important cause of response variability to clopidogrel therapy. Cigarette smoking induces cytochrome P450 (31), which is involved in clopidogrel metabolism. In addition, smoking also influences the long-term benefit of clopidogrel therapy in PCI patients (19). In our study, CYP2C19 genotyping could better predict future clinical events in current smokers following PCI procedures.
In 2014, Tabata et al. found that kidney function status modified the effects of CYP2C19 polymorphisms on clinical outcome in patients following coronary stent implantation (21). Including 154 CKD patients and 177 non-CKD patients, CYP2C19 LOF allele carriers were identified as independent cardiovascular event predictors only in the non-CKD group. CKD is also a prognostic factor in PCI patients. Moreover, previous clinical observations suggest that CKD itself contributes to high residual platelet reactivity. Thus, the CKD contribution to platelet reactivity and adverse clinical events might outweigh the influence of the CYP2C19 genotype in CKD patients.

Higher rates of cardiovascular events were predicted by CYP2C16 genotype in other populations in our study, including males, age $>60$ years, BMI $>24 \mathrm{~kg} / \mathrm{m}^{2}$, and SYNTAX score $>15$. Differing adverse event rates might explain some previous controversies regarding the effects of CYP2C19 LOF alleles on clinical outcome in patients receiving DAPT. In patients with traditional high-risk predictors (male, aged, high SYNTAX score, high BMI), the adverse event incidence increased, causing a more significant difference of clinical outcomes among genotype subgroups than in the low-risk population. In other words, using CYP2C19 genotypes is more suitable in populations with high adverse cardiovascular event risk.

Increasing evidence indicates that East Asian patients have different risk profiles for thrombophilia and bleeding compared with Caucasian patients. Thus, a different 'therapeutic window' of antiplatelet therapy might be appropriate in East Asian patients (32-35). The CYP2C19 LOF genotype is almost twice as prevalent in Asian populations compared to Caucasian populations, thus contributing to the high prevalence of low clopidogrel responsiveness in Asians (36). However, the incidence of adverse ischemic outcomes or stent thrombosis after PCI is similar or lower than in white patients, and is called the "East Asian paradox" (33). Our study findings indicate that in East Asian PCI patients, CYP2C19 genotypes might be an effective tool for predicting clinical outcomes and selecting an appropriate intensity of antiplatelet therapy.

There were several limitations in the present study. First, as showed in Figure 1, only about 800 patients of the total PCI cohort took genotype test, leading to the unavoidable selection bias of the present study. This limits its homogeneity in the general PCI population Second, the individual variability in clopidogrel responsiveness was multifactorial. CYP2C19*2 explained only about $12 \%$ of the 
potential variation in clopidogrel responses. Other potential genetic mutations were not included in our research, such as CYP2C19*17, PON1, and ABCB1. Third, this is a singlecenter study with a limited sample size. Although a singlecenter design helps maintain standard procedures (PCI, genotype, medication and follow-up), a multi-center design with a larger sample could provide more replicable data. Finally, more than $70 \%$ of subjects in this study were male. This gender disparity might lead to a relatively inadequate statistical power in female subgroups.

\section{Conclusions}

Our results indicate that CYP2C19 genotype has a greater prognostic value in PCI patients with one of the following clinical characteristics: male, age $>60$ years, BMI $>24 \mathrm{~kg} / \mathrm{m}^{2}$, SYNTAX score $>15$, current smoking, and no chronic kidney disease. The study findings support the idea that a personalized approach using CYP2C19 genotyping to guide post-PCI antiplatelet therapy might be appropriate.

\section{Acknowledgments}

Funding: This work was supported by the Chinese Academy of Medical Sciences Innovation Fund for Medical Sciences (CIFMS 2016-I2M-1-009) and the National Natural Science Foundation of China (No. 81800327; No. 81825003).

\section{Footnote}

Reporting Checklist: The authors have completed the STROBE reporting checklist Available at http://dx.doi. org/10.21037/atm-20-7724

Data Sharing Statement: Available at http://dx.doi. org/10.21037/atm-20-7724

Conflicts of Interest: All authors have completed the ICMJE uniform disclosure form (available at http://dx.doi. org/10.21037/atm-20-7724). The authors have no conflicts of interest to declare.

Ethical Statement: The authors are accountable for all aspects of the work in ensuring that questions related to the accuracy or integrity of any part of the work are appropriately investigated and resolved. All the participants provided signed informed consent for the original cohort study and subsequent analysis. The study was conducted in accordance with the Declaration of Helsinki (as revised in 2013). The study was approved by institutional ethics committee of Fuwai Hospital (No. 2016-847) and individual consent for this retrospective analysis was waived.

Open Access Statement: This is an Open Access article distributed in accordance with the Creative Commons Attribution-NonCommercial-NoDerivs 4.0 International License (CC BY-NC-ND 4.0), which permits the noncommercial replication and distribution of the article with the strict proviso that no changes or edits are made and the original work is properly cited (including links to both the formal publication through the relevant DOI and the license). See: https://creativecommons.org/licenses/by-nc-nd/4.0/.

\section{References}

1. Gurbel PA, Tantry US. Combination antithrombotic therapies. Circulation 2010;121:569-83.

2. Gurbel PA, Bliden KP, Hiatt BL, et al. Clopidogrel for coronary stenting: response variability, drug resistance, and the effect of pretreatment platelet reactivity. Circulation 2003;107:2908-13.

3. Hulot JS, Bura A, Villard E, et al. Cytochrome P450 2C19 loss-of-function polymorphism is a major determinant of clopidogrel responsiveness in healthy subjects. Blood 2006;108:2244-7.

4. Trenk D, Hochholzer W, Fromm MF, et al. Cytochrome P450 2C19 681G>A polymorphism and high onclopidogrel platelet reactivity associated with adverse 1-year clinical outcome of elective percutaneous coronary intervention with drug-eluting or bare-metal stents. J Am Coll Cardiol 2008;51:1925-34.

5. Yamamoto K, Hokimoto S, Chitose T, et al. Impact of CYP2C19 polymorphism on residual platelet reactivity in patients with coronary heart disease during antiplatelet therapy. J Cardiol 2011;57:194-201.

6. Mega JL, Close SL, Wiviott SD, et al. Cytochrome p-450 polymorphisms and response to clopidogrel. $\mathrm{N} \mathrm{Engl} \mathrm{J}$ Med 2009;360:354-62.

7. Tang YD, Wang W, Yang $M$, et al. Randomized Comparisons of Double-Dose Clopidogrel or Adjunctive Cilostazol Versus Standard Dual Antiplatelet in Patients With High Posttreatment Platelet Reactivity: Results of the CREATIVE Trial. Circulation 2018;137:2231-2245.

8. Bouman HJ, Schomig E, van Werkum JW, et al. Paraoxonase-1 is a major determinant of clopidogrel 
efficacy. Nat Med 2011;17:110-6.

9. Simon T, Verstuyft C, Mary-Krause M, et al. Genetic determinants of response to clopidogrel and cardiovascular events. N Engl J Med 2009;360:363-75.

10. Sibbing D, Stegherr J, Latz W, et al. Cytochrome P450 2C19 loss-of-function polymorphism and stent thrombosis following percutaneous coronary intervention. Eur Heart J 2009;30:916-22.

11. Collet JP, Hulot JS, Pena A, et al. Cytochrome P450 2C19 polymorphism in young patients treated with clopidogrel after myocardial infarction: a cohort study. Lancet 2009;373:309-17.

12. Jeong YH, Tantry US, Kim IS, et al. Effect of CYP2C19*2 and *3 loss-of-function alleles on platelet reactivity and adverse clinical events in East Asian acute myocardial infarction survivors treated with clopidogrel and aspirin. Circ Cardiovasc Interv 2011;4:585-94.

13. Li X, Yu C, Wang T, et al. Effect of cytochrome P450 2C19 polymorphisms on the clinical outcomes of voriconazole: a systematic review and meta-analysis. Eur J Clin Pharmacol 2016;72:1185-1193.

14. Pare G, Mehta SR, Yusuf S, et al. Effects of CYP2C19 genotype on outcomes of clopidogrel treatment. N Engl J Med 2010;363:1704-14.

15. Li C, Zhang L, Wang $\mathrm{H}$, et al. Gene variants in responsiveness to clopidogrel have no impact on clinical outcomes in Chinese patients undergoing percutaneous coronary intervention - A multicenter study. Int J Cardiol 2017;240:360-6.

16. Gurbel PA and Tantry US. Do platelet function testing and genotyping improve outcome in patients treated with antithrombotic agents?: platelet function testing and genotyping improve outcome in patients treated with antithrombotic agents. Circulation 2012;125:1276-87; discussion 1287.

17. Tantry US, Kereiakes DJ, Gurbel PA. Clopidogrel and proton pump inhibitors: influence of pharmacological interactions on clinical outcomes and mechanistic explanations. JACC Cardiovasc Interv 2011;4:365-80.

18. Park Y, Jeong YH, Tantry US, et al. Accelerated platelet inhibition by switching from atorvastatin to a nonCYP3A4-metabolized statin in patients with high platelet reactivity (ACCEL-STATIN) study. Eur Heart J 2012;33:2151-62.

19. Gurbel PA, Bliden KP, Logan DK, et al. The influence of smoking status on the pharmacokinetics and pharmacodynamics of clopidogrel and prasugrel: the PARADOX study. J Am Coll Cardiol 2013;62:505-12.
20. Sibbing D, von Beckerath O, Schomig A, et al. Impact of body mass index on platelet aggregation after administration of a high loading dose of $600 \mathrm{mg}$ of clopidogrel before percutaneous coronary intervention. Am J Cardiol 2007;100:203-5.

21. Tabata N, Hokimoto S, Akasaka T, et al. Chronic kidney disease status modifies the association of CYP2C19 polymorphism in predicting clinical outcomes following coronary stent implantation. Thromb Res 2014;134:939-44.

22. Arima Y, Hokimoto S, Akasaka T, et al. Comparison of the effect of CYP2C19 polymorphism on clinical outcome between acute coronary syndrome and stable angina. J Cardiol 2015;65:494-500.

23. Cutlip DE, Windecker S, Mehran R, et al. Clinical end points in coronary stent trials: a case for standardized definitions. Circulation 2007;115:2344-51.

24. Mehran R, Rao SV, Bhatt DL, et al. Standardized bleeding definitions for cardiovascular clinical trials: a consensus report from the Bleeding Academic Research Consortium. Circulation 2011;123:2736-47.

25. Notarangelo FM, Maglietta G, Bevilacqua P, et al. Pharmacogenomic Approach to Selecting Antiplatelet Therapy in Patients With Acute Coronary Syndromes: The PHARMCLO Trial. J Am Coll Cardiol 2018;71:1869-77.

26. Angiolillo DJ, Capodanno D, Danchin N, et al. Derivation, Validation, and Prognostic Utility of a Prediction Rule for Nonresponse to Clopidogrel: The ABCD-GENE Score. JACC Cardiovasc Interv 2020;13:606-617.

27. Saito $Y$, Nishi T, Wakabayashi S, et al. Validation of the ABCD-GENE score to identify high platelet reactivity in east Asian patients undergoing percutaneous coronary intervention. Int J Cardiol 2021;327:15-8.

28. Claassens DMF, Vos GJA, Bergmeijer TO, et al. A Genotype-Guided Strategy for Oral P2Y12 Inhibitors in Primary PCI. N Engl J Med 2019;381:1621-31.

29. Pereira NL, Farkouh ME, So D, et al. Effect of GenotypeGuided Oral P2Y12 Inhibitor Selection vs Conventional Clopidogrel Therapy on Ischemic Outcomes After Percutaneous Coronary Intervention: The TAILOR-PCI Randomized Clinical Trial. JAMA 2020;324:761-771.

30. Bliden KP, Dichiara J, Lawal L, et al. The association of cigarette smoking with enhanced platelet inhibition by clopidogrel. J Am Coll Cardiol 2008;52:531-3.

31. Zevin S, Benowitz NL. Drug interactions with tobacco smoking. An update. Clin Pharmacokinet 1999;36:425-38.

32. Tantry US, Bonello L, Aradi D, et al. Consensus and update on the definition of on-treatment platelet reactivity 
to adenosine diphosphate associated with ischemia and bleeding. J Am Coll Cardiol 2013;62:2261-73.

33. Jeong YH. "East asian paradox": challenge for the current antiplatelet strategy of "one-guideline-fits-all races" in acute coronary syndrome. Curr Cardiol Rep 2014;16:485.

34. Levine GN, Jeong YH, Goto S, et al. Expert consensus document: World Heart Federation expert consensus statement on antiplatelet therapy in East Asian patients with ACS or undergoing PCI. Nat Rev Cardiol 2014;11:597-606.

35. Mak KH, Bhatt DL, Shao M, et al. Ethnic

Cite this article as: Wang W, Shao C, Xu B, Wang J, Yang M, Chen J, Zhang K, Wang S, Li P, Tang YD. CYP2C19 genotype has prognostic value in specific populations following coronary stenting. Ann Transl Med 2021;9(13):1066. doi: 10.21037/atm$20-7724$ variation in adverse cardiovascular outcomes and bleeding complications in the Clopidogrel for High Atherothrombotic Risk and Ischemic Stabilization, Management, and Avoidance (CHARISMA) study. Am Heart J 2009;157:658-65.

36. Man M, Farmen M, Dumaual C, et al. Genetic variation in metabolizing enzyme and transporter genes: comprehensive assessment in 3 major East Asian subpopulations with comparison to Caucasians and Africans. J Clin Pharmacol 2010;50:929-40. 